\title{
DICHOTOMY IN THE DYNAMICAL STATUS OF MASSIVE CORES IN ORION
}

\author{
T. Velusamy, ${ }^{1}$ R. Peng, ${ }^{2}$ D. Li, ${ }^{1}$ P. F. Goldsmith, ${ }^{1}$ and William D. Langer ${ }^{1}$ \\ Received 2008 September 2; accepted 2008 October 8; published 2008 October 21
}

\begin{abstract}
To study the evolution of high-mass cores, we have searched for evidence of collapse motions in a large sample of starless cores in the Orion molecular cloud. We used the Caltech Submillimeter Observatory telescope to obtain spectra of the optically thin $\left(\mathrm{H}^{13} \mathrm{CO}^{+}\right)$and optically thick $\left(\mathrm{HCO}^{+}\right)$high-density tracer molecules in 27 cores with masses $>1 M_{\odot}$. The red- and blue-asymmetries seen in the line profiles of the optically thick line with respect to the optically thin line indicate that $2 / 3$ of these cores are not static. We detect evidence for infall (inward motions) in 9 cores and outward motions for 10 cores, suggesting a dichotomy in the kinematic state of the nonstatic cores in this sample. Our results provide an important observational constraint on the fraction of collapsing (inward motions) versus noncollapsing (reexpanding) cores for comparison with model simulations.
\end{abstract}

Subject headings: ISM: clouds — ISM: individual (Orion) — ISM: kinematics and dynamics —

ISM: molecules - stars: formation

\section{INTRODUCTION}

In giant molecular clouds, the formation of massive cold molecular cores can be induced by gravoturbulent cloud fragmentation (cf. Mac Low \& Klessen 2004). McKee \& Tan (2003) envisage that these cores are molecular condensations in a turbulence-supported quasi-equilibrium state, which ultimately form a single protostar or a gravitationally bound cluster of massive protostars. This important stage in star formation is not well understood due to lack of observational data on prestellar cores on the verge of collapse. To study this stage we obtained new data on the kinematic state for a sizable number of Orion cores. Li et al. (2007, hereafter Paper II) identified 51 dust cores in a $350 \mu \mathrm{m}$ submillimeter continuum survey of the quiescent regions of the Orion molecular cloud using the SHARC II camera at the Caltech Submillimeter Observatory (CSO). A combination of the enhanced spatial resolution analysis of the submillimeter data using the HiRes deconvolution tool (see Velusamy et al. 2008) and our knowledge of the temperature from the ammonia mapping by $\mathrm{Li}$ et al. (2003, hereafter Paper I) enabled us to determine relatively accurately the key physical parameters (including size and mass) of cores in this representative sample of starless cores in a highmass star forming cloud. This Orion core sample is a collection of resolved or nearly resolved cores, with a mean mass of 9.8 $M_{\odot}$, which is 1 order of magnitude higher than that of resolved cores in low-mass star-forming regions. The majority of the cores appear to be too massive to be thermally supported as BonnerEbert spheres for $T_{K}<30 \mathrm{~K}$ (Paper II), or even with turbulence $\sim 1.5 \mathrm{~km} \mathrm{~s}^{-1}$ (for reference the mean line width observed in these cores is $0.78 \mathrm{~km} \mathrm{~s}^{-1}$ ). Therefore, magnetic support may be important in these cores (cf. Mouschovias 1991). Here we present new spectroscopic observations to determine the kinematics of these cores. We discuss the prevalence of dynamically unstable high-mass cores in this sample and the equally large proportion of cores showing either collapse or expansion.

\section{OBSERVATIONS}

We selected a subset of 27 cores from the sample in Paper II which have mass $>1 M_{\odot}$. The observations were made at the 10.4

\footnotetext{
${ }^{1}$ Jet Propulsion Laboratory, California Institute of Technology, 4800 Oak Grove Drive, Pasadena, CA 91109; Velusamy@jpl.nasa.gov.

${ }^{2}$ Caltech Submillimeter Observatory, 111 Nowelo Street, Hilo, HI 96720; peng@submm.caltech.edu.
}

m telescope of the Caltech Submillimeter Observatory (CSO) at Mauna Kea, Hawaii, in 2007 March and December. The cores and their coordinates were selected from Table 1 in Paper II. We observed both $\mathrm{HCO}^{+}(3-2)$ at $267.557620 \mathrm{GHz}$, and $\mathrm{H}^{13} \mathrm{CO}^{+}(3-$ 2) at $260.255478 \mathrm{GHz}$ in all 27 cores. We used the $230 \mathrm{GHz}$ band heterodyne receiver and the 1024 channel acousto-optic spectrometer with a bandwidth of $50 \mathrm{MHz}$. The FWHM antenna beam was $26^{\prime \prime}$ and the main-beam efficiency was 0.66 . To improve the $\mathrm{S} / \mathrm{N}$ on the optically thin $\mathrm{H}^{13} \mathrm{CO}^{+}(3-2)$ spectra, 3 adjacent velocity channels were co-added. The velocity $V_{0}$ and line width $\Delta V_{\text {thin }}$ for each core were obtained using Gaussian profile fit to the optically thin $\mathrm{H}^{13} \mathrm{CO}^{+}(3-2)$ lines. The spectra of all 27 cores are shown in Figure 1. The spectra of the cores are presented in three groups as a function of their asymmetry (see $\S 3$ ) in the $\mathrm{HCO}^{+}$line with (a) blueshifted peak emission, $(b)$ redshifted peak emission, and (c) no significant asymmetry with respect to the $\mathrm{H}^{13} \mathrm{CO}^{+}$spectrum. The spectra within each group are arranged in order of their masses.

For most of the cores the line widths for $\mathrm{H}^{13} \mathrm{CO}^{+}$are $>0.5$ $\mathrm{km} \mathrm{s}^{-1}$ and appear to be dominated by turbulence compared to $\mathrm{HCO}^{+}$thermal line widths $<0.2 \mathrm{~km} \mathrm{~s}^{-1}$ for $T_{K}<20 \mathrm{~K}$. Thus the majority of our cores are supersonic. Using the total line width as an upper limit of the equivalent sound speed we estimated approximate Bonnor-Ebert (B-E) critical mass $\left(M_{\mathrm{B}-\mathrm{E}}\right)$ for each core (see Paper II). Only 3 cores have masses within a factor 2.5 of their critical masses while the other cores all have masses well above their critical masses for stability.

\section{RESULTS AND DISCUSSION}

To study the kinematic status of the cores we use the spectral line asymmetry as observed between a pair of optically thick and thin molecular transitions. We define the red- and blue-asymmetry by the peak emission in the optically thick line appearing redward and blueward, respectively, with respect to the peak in the optically thin spectrum. The method of spectral line asymmetry (Leung \& Brown 1977; Zhou 1992) has commonly been used to identify collapse candidates. Under certain assumed collapse conditions (e.g., Shu et al. 1987) an optically thick line [e.g., $\left.\mathrm{HCO}^{+}(3-2)\right]$ in a collapsing core shows a blue-asymmetry in the line profile observed toward the collapse center, while an optically thin line [e.g., $\left.\mathrm{H}^{13} \mathrm{CO}^{+}(3-2)\right]$ appears symmetric. Gregersen et al. (1997) have used the same pair of lines to search for infall in 19 lowmass cores. They detected blue-asymmetry in 9 Class 0 objects, and red-asymmetry in 2 objects. Using the $\mathrm{HCO}^{+}(3-2)$ line as the 

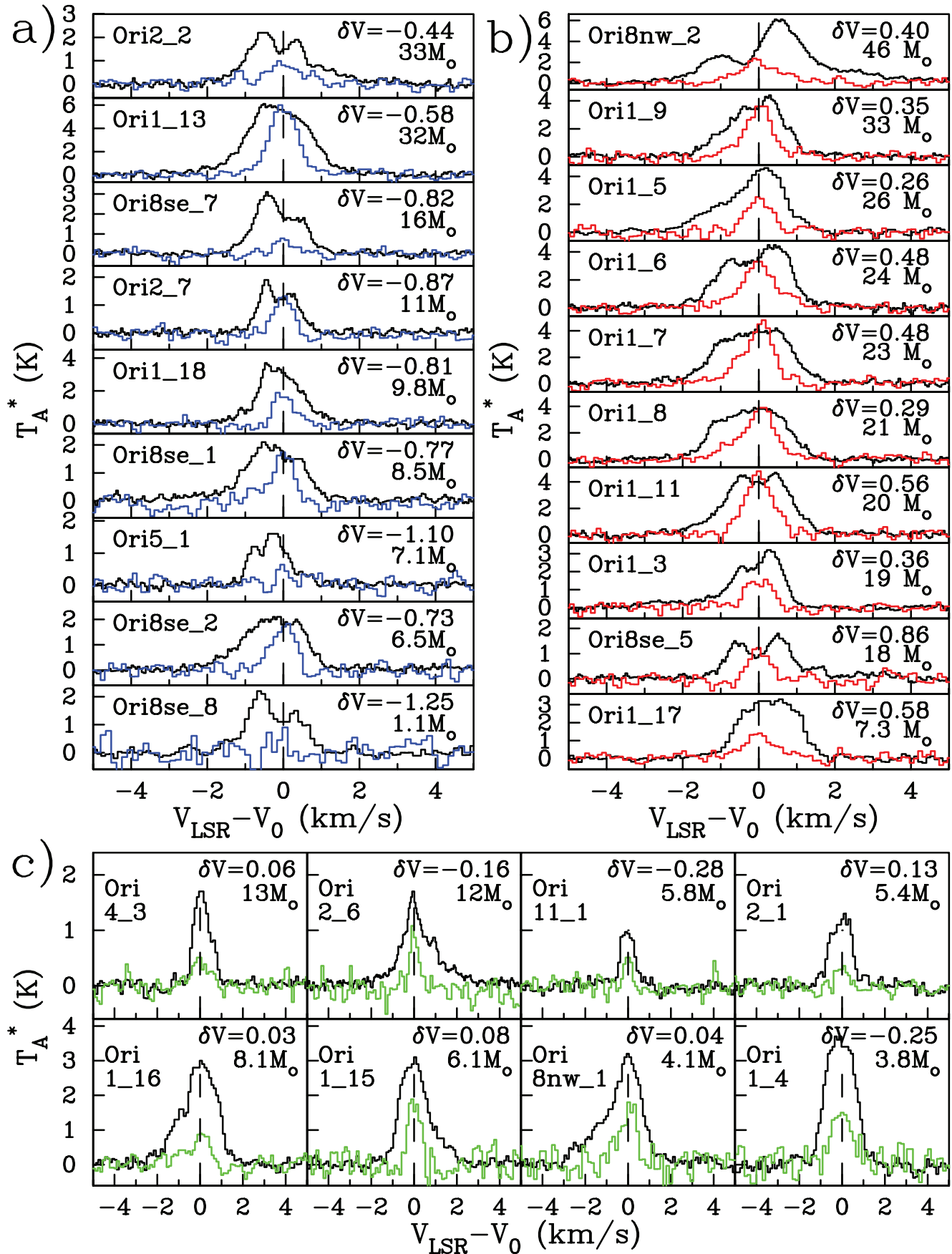

FIG. 1.- Spectra of the optically thick $\left[\mathrm{HCO}^{+}(3-2)\right]$ and thin $\left[\mathrm{H}^{13} \mathrm{CO}^{+}(3-2)\right]$ lines for 27 cores in our sample with mass $>1 M_{\odot}$. The $\mathrm{HCO}^{+}$spectra are shown in black and the $\mathrm{H}^{13} \mathrm{CO}^{+}$spectra in color. The $\mathrm{H}^{13} \mathrm{CO}^{+}$intensities have been multiplied by a factor of 3 . The channel velocities in each panel are $V_{L S R}-V_{0}$ where $V_{0}$ is the peak velocity of $\mathrm{H}^{13} \mathrm{CO}^{+}$profile. The mass of the core and the asymmetry parameter $\delta V$ (see eq. [1]) are indicated. The cores are identified as given in Paper II. The spectra are grouped into cores with $(a)$ blue asymmetry, $(b)$ red asymmetry, and $(c)$ no significant asymmetry.

optically thick tracer, in their search for infall candidates in lowmass cores, Mardones et al. (1997) detected blue-asymmetry in 8 , red-asymmetry in 3, and found one (VLA 1623) to be inconclusive. Fuller et al. (2005) observed 77 submillimeter sources associated with a sample of IRAS-selected objects, all believed to be high-mass protostellar objects. They identified 22 infall candidates none of which show any red-asymmetry. Thus red-asymmetries are rare in low-mass cores (cf. Lee et al. 2001) as well as in high-mass cores containing protostars and the few cores showing red-asymmetries are caused by outflows (Gregersen et al. 1997). In contrast, in our Orion sample of high-mass starless cores (not containing any known protostars) approximately $2 / 3$ show blueor red-asymmetries, roughly in equal numbers.
In principle, information on the velocity structure $v(r)$ within the core is contained in the line profile averaged over the core. In the spectra shown in Figure 1, the line-of-sight motions within the core produce Doppler shifts and features in the line profiles averaged over the core. A static spherical core displays a symmetric spectral line profile for low optical depths. At large optical depths it exhibits a self-absorption dip at the center with symmetric blue and red peaks. Radial variations in physical conditions, e.g., temperature, density, molecular abundances, and line widths, do not affect the symmetry of the observed spectrum relative to the central velocity of the core. By looking at whether the line emission is stronger in the red or blue, one can distinguish between infall and expansion in the core (Leung \& Brown 1977; Zhou et al. 1993). 
Schematically, in the case of radial infall the red and blue emissions come from, respectively, the hemispheres closer to and farther from the observer. If the excitation temperature $\left(T_{\mathrm{ex}}\right)$ decreases radially outward from the core center, as expected for $\mathrm{HCO}^{+}(3-$ 2 ), the line of sight from the observer at redshifted frequencies first encounters cooler gas (at greater distance from the core center), while at blueshifted frequencies warmer gas (closer to the core center) is encountered first. Therefore, subject to the caveat on the velocity field discussed below, the blue emission is stronger than the red, producing a blue-asymmetry. In the case of radial expansion of the core the blue and red hemispheres are reversed and the red emission is stronger, resulting in a red-asymmetry.

The effects of the self-absorption on the red or blue peaks of the spectrum depends critically on the velocity field as a function of radius. There is no self-absorption in the peaks if $v(r) \propto r$, as every line-of-sight velocity component corresponds to a single point along the line of sight. However, for a velocity field having a power law with negative index, for example, $v(r) \propto r^{-0.5}$, as in the case of inside-out collapse model (Shu et al. 1987), every lineof-sight velocity component corresponds to two points at different distances from the core center (see Fig. 8 in Zhou et al. 1993). Thus this velocity law favors a stronger self-absorption in the red as the two isovelocity points in the red hemisphere correspond to a high and low $T_{\text {ex }}$, respectively, farther and closer to the observer (see above). To produce the red- and blue-asymmetries by selfabsorption, we require a favorable radial variation of not only velocity, $v(r)$, but also excitation temperature, $T_{\text {ex }}(r)$. In a region where the $\mathrm{HCO}^{+}$emission is produced, the excitation of $\mathrm{HCO}^{+}(3-$ 2 ) is subthermal and is dominated by the radial increase in density toward the core center. Thus $T_{\mathrm{ex}}(r)$ will always increase radially toward the center essentially independent of the temperature profile (Paper I). LVG model calculations show that such conditions in a family of model clouds with density $\sim 10^{5}-10^{6} \mathrm{~cm}^{-3}$ and $T_{K} \sim$ $15-25 \mathrm{~K}$ can produce an infall blue-asymmetry. We can rule out rotation as the cause of asymmetry in these cores as they are spatially unresolved and spectra were obtained toward the center of rotation of the cores (Menten et al. 1987; Adelson \& Leung 1988).

Clearly, the asymmetries observed in the spectra in Figure 1 show that a majority of these cores (19 out of 27) are not static but have inward or outward motions. The interpretation of the blue-asymmetry as infall is well understood and has been modeled extensively (e.g., Choi et al. 1995). The interpretation of the redasymmetry as outward expansion is less obvious. We can rule out interpreting red-asymmetry as infall because it requires abnormal conditions in which the excitation temperature will be lower near the core center. On the other hand, a reasonable interpretation of red-asymmetry is outward (expansion) motion in the core with a velocity law $v(r) \propto r^{-\beta}$ where $\beta>0$. Although a detailed interpretation of the dynamics associated with asymmetries in the line profiles will be model dependent, considering the above scenarios, here we assume that the cores showing red- or blue-asymmetries have either inward or outward motions. Among the 27 cores for which we have both the optically thick and thin spectral line profiles, 9 show blue-asymmetry, 10 show red-asymmetry, while the remaining 8 have roughly symmetrical profiles with a single velocity peak. Following Mardones et al. (1997) we quantify the red- and blue-asymmetry by the asymmetry parameter:

$$
\delta V=\left(V_{\text {thick }}-V_{\text {thin }}\right) / \Delta V_{\text {thin }},
$$

where $V_{\text {thick }}$ is the velocity of the peak of the opaque $\left(\mathrm{HCO}^{+}\right)$ line, $V_{\text {thin }}$ is the velocity of the peak of the optically thin $\left(\mathrm{H}^{13} \mathrm{CO}^{+}\right)$line, and $\Delta V_{\text {thin }}$ is the line width of the thin line; $\delta V$ is indicated on each spectra in Figure 1. In Figure $2 a$ we show the distribution of the observed asymmetry parameters. The distribution is bimodal with peaks having distinct blue( $\delta V$ negative) and red- ( $\delta V$ positive) asymmetries.

A fairly clear demarcation between the red- and blue-asymmetries as a function of core mass is evident in Figure $2 b$. The higher mass cores (with the exception of Ori 1_13 and Ori 2_2) tend to show a red-asymmetry while the lower mass cores show a blue-asymmetry. If we interpret the red-asymmetry as outward motion (expansion) within the core it is conceivable that these cores will fragment and eventually form only multiple lower mass stars. At the high end of the mass distribution, all of the 12 cores, having masses $>15 M_{\odot}$, show either red- or blue-asymmetry and are either collapsing ( 3 cores) or expanding ( 9 cores). Thus for the high-mass cores $\left(>15 M_{\odot}\right.$ ) we observe a larger proportion (by a factor 3 ) of cores showing expansion compared to those showing collapse. In contrast, among the 16 lower mass cores $\left(<15 M_{\odot}\right)$ only one (Ori 1_17) shows evidence for expansion, while 6 cores show collapse and 8 are static. Assuming the cores showing blue-asymmetry will eventually form stars (3/12 for $>15 M_{\odot}$ and $6 / 16$ for $\left.<15 M_{\odot}\right)$, we can estimate that $1 / 3$ of the cores $(\sim 32 \%$ of the total mass in our present sample of 27 cores in Orion) will form high-mass stars.

Our Orion sample represents a population of massive molecular cloud cores in which the majority of the cores are dynamic out-ofequilibrium structures. We detect cores in roughly equal proportion showing all three kinematic states: static, outward, and inward motion. Therefore, we may be seeing, statistically, a snapshot of a population of cores exhibiting oscillatory motion. Oscillations in cores about a stable equilibrium configuration, producing the redand blue-asymmetries, have been observed in low-mass cores (Redman et al. 2006; Keto et al. 2006; Broderick et al. 2007). The masses of our cores, with the exception of a few, far exceed the critical masses for equilibrium as isothermal Bonnor-Ebert spheres (see Paper II, Fig. 8). However, it is conceivable that they are magnetically supported. Although they are thermally supercritical, they could still be magnetically subcritical having equilibrium configurations (cf. Mouschovias 1991). Under such conditions they are easily excited by the external interstellar medium to exhibit oscillatory behavior, consistent with the distribution of the kinetic state of cores observed in our sample. The majority of cores above the median mass of our sample have red asymmetric profiles indicative of expansion. $\mathrm{Nu}-$ merical models also suggest that massive molecular cloud cores are in general likely to be dynamic, out-of-equilibrium structures, rather than quasi-hydro/magnetostatic structures (Vázquez-Semadeni et al. 2005). In their models for the evolution of clumps and cores formed as turbulent density fluctuations, the dynamically compressed regions must either proceed to collapse right away or reexpand. These models predict that not all cores observed in molecular clouds will necessarily form stars and that a class of failed cores should exist, which will eventually redisperse. Thus we should see some fraction of cores displaying outward as well as inward motions. Our results do show a larger detection of kinematic signatures with inward and outward motions than in previous studies, particularly in low-mass starless cores (Lee et al. 2001). This result may not be unique to Orion and in general may be more common in massive than in low-mass cores, but we are better able to detect kinematic signatures in Orion due to its proximity. Our results provide an important observational constraint on the fraction of collapsing (inward motions) versus noncollapsing (reexpanding) cores for comparison with model simulations.

We thank Konstantinos Tassis for comments on the dynamics of magnetically supported cores. We thank Jorge Pineda for running cloud models incorporating specific large-scale velocity fields to confirm the expected behavior of the red- and blueasymmetries in the Orion cores. The research described in this 


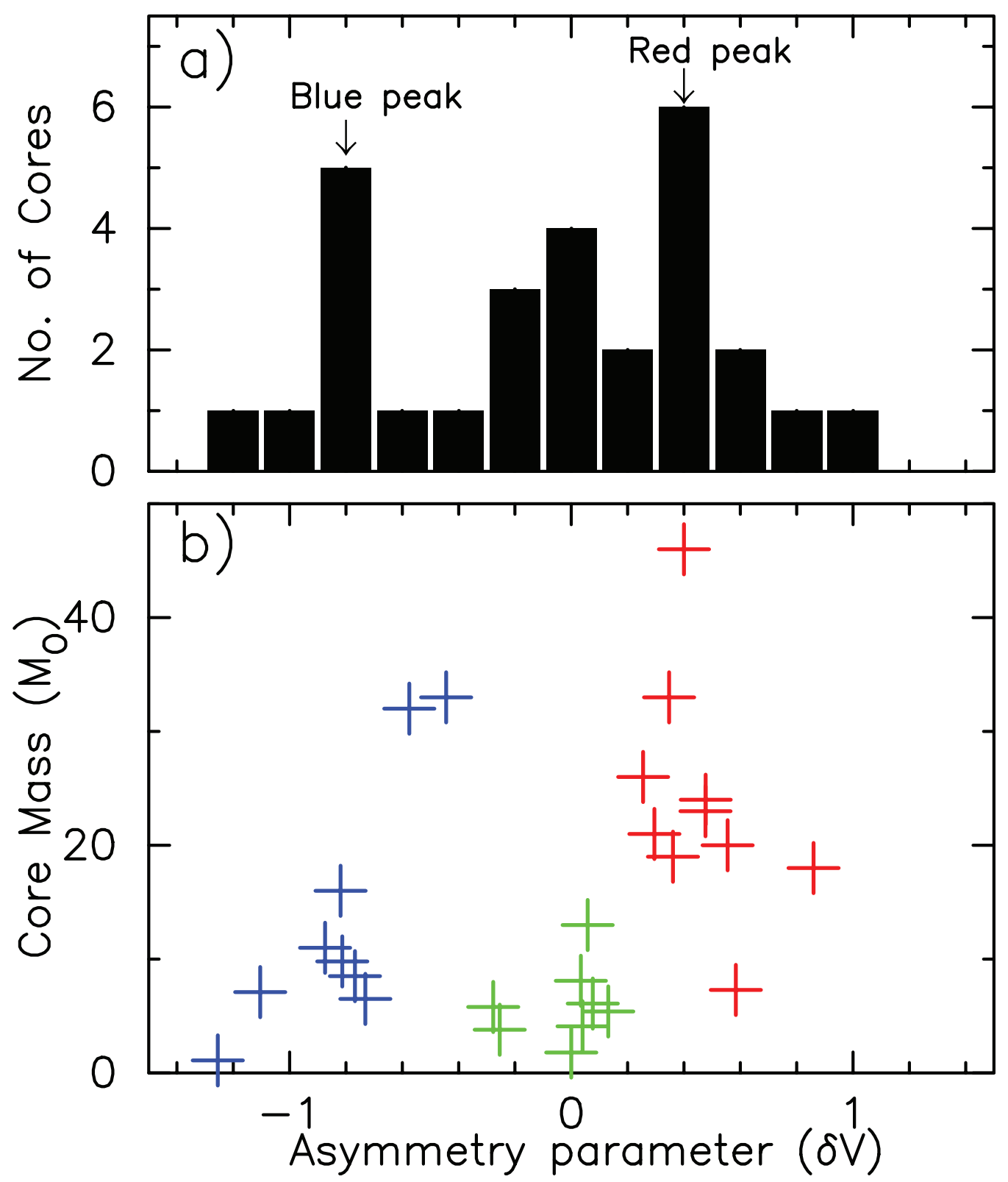

FIG. 2.- (a) Distribution of the velocity asymmetry parameter $\delta V$ defined in eq. (1) for the full sample of 27 cores obtained using optically thick $\left(\mathrm{HCO}^{+}\right)$and thin $\left(\mathrm{H}^{13} \mathrm{CO}^{+}\right)$lines. Note the dichotomy in the kinematic state of the nonstatic high-mass cores. $(b)$ Asymmetry parameter vs. core mass. The blue and red crosses correspond to cores showing blue- and red-peak asymmetry, respectively, in $\mathrm{HCO}^{+}$with respect to $\mathrm{H}^{13} \mathrm{CO}^{+}$, as shown in Figs. $1 a$ and $1 b$. The green crosses represent cores (Fig. 1c) that do not show any significant asymmetry. Note that the large proportion of the high-mass cores have a red asymmetry.

Letter was carried out at the Jet Propulsion Laboratory, California Institute of Technology, under a contract with the $\mathrm{Na}$ tional Aeronautics and Space Administration and supported by a grant from NASA Astronomy and Physics Research and Analysis Program. Research at the Caltech Submillimeter Observatory is supported by NSF grant AST-0229008.

\section{REFERENCES}

Adelson, L. M., \& Leung, C. M. 1988, MNRAS, 235, 349

Broderick, A. E., Keto, E., Lada, C. J., \& Narayan, R. 2007, ApJ, 671, 1832

Choi, M., Evans, N. J., II, Gregersen, E. M., \& Wang, Y. 1995, ApJ, 448, 742

Fuller, G. A., Williams, S. J., \& Sridharan, T. K. 2005, A\&A, 442, 949

Gregersen, E. M., Evans, N. J., II, Zhou, S., \& Choi, M. 1997, ApJ, 484, 256

Keto, E., Broderick, A., Lada, C. J., \& Narayan, R. 2006, ApJ, 652, 1366

Lee, C. W., Myers, P. C., \& Tafalla, M. 2001, ApJS, 136, 703

Leung, C. M., \& Brown, R. L. 1977, ApJ, 214, L73

Li, D., Goldsmith, P. F., \& Menten, K. 2003, ApJ, 587, 262 (Paper I)

Li, D., et al. 2007, ApJ, 655, 351 (Paper II)

Mac Low M.-M., \& Klessen, R. S. 2004, Rev. Mod. Phys., 76, 125
Mardones, D., et al. 1997, ApJ, 489, 719

McKee, C. F., \& Tan, J. C. 2003, ApJ, 585, 850

Menten, K. M., Serabyn, E., Gusten, R., \& Wilson, T. L. 1987, A\&A, 177, L57

Mouschovias, T. Ch. 1991, ApJ, 373, 169

Redman, M. P., Keto, E., \& Rawlings, J. M. C. 2006, MNRAS, 370, L1

Shu, F. H., Adams, F. C., \& Lizano, S. 1987, ARA\&A, 25, 23

Vázquez-Semadeni, E., et al. 2005, ApJ, 618, 344

Velusamy, T., et al. 2008, AJ, 136, 197

Zhou, S. 1992, ApJ, 394, 204

Zhou, S., Evans, N. J., II, Kompe, C., \& Walmsley, C. M. 1993, ApJ, 404, 232 